\title{
MOVILIDADES Y PRÁCTICAS SOCIOESPACIALES FRONTERIZAS ENTRE ARICA Y TACNA. DEL SENTIDO DE FRONTERA A LA TRANSFRONTERICIDAD ENTRE CIUDADES*
}

\author{
SOCIAL AND SPACE BORDER MOVING AND PRACTICES BETWEEN ARICA \\ AND TACNA. FROM THE SENSE OF FRONTIER TO TRANSFRONTERICIDAD \\ BETWEEN CITIES
}

\author{
Yasna Contreras ${ }^{* *}$, Marcela Tapia $^{* * *}$ y Nannette Liberona***
}

\begin{abstract}
El estudio de la movilidad entre ciudades fronterizas tensiona las nociones de frontera y discute las ideas que por largo tiempo se han utilizado en los estudios migratorios. Así las prácticas sociales fronterizas demuestran que la transfrontericidad de dos ciudades (Arica y Tacna) están dadas fundamentalmente por el afán de los habitantes de uno y otro lado de aprovechar las ventajas del cruce, dando lugar a distintos tipos de movilidades. Las disparidades y asimetrías entre ciudades y el notorio sentido de frontera no impide que las personas crucen diaria, semanal o mensualmente para trabajar o comerciar en Arica o abaratar el costo de la vida, visitar al médico o disfrutar de la gastronomía y el ocio en Tacna. De modo que la movilidad en la frontera y las prácticas socioespaciales como continuidad espacio-temporal son la base de la constitución de Arica y Tacna como Complejos Urbanos Transfronterizos.

Palabras claves: Migración, prácticas espaciales, frontera y movilidad.
\end{abstract}

The study of human mobility between border cities strains notions of border and calls into question ideas that have long been used in migration studies. Border social practices show that the transborder nature of two cities (Arica and Tacna) are mainly the result of their inhabitants' desire to take advantage of the act of crossing, which leads to various types of mobilities. The disparities and asymmetries of the cities and strong sense of border that exists there do not prevent people from crossing daily, weekly or monthly to work or sell goods in Arica or decrease the cost of living, seek medical care or enjoy cuisine and recreational activities in Tacna. As such, mobility at the border and socio-spatial practices as well as space-time continuity form the basis of the constitution of Arica and Tacna as Transborder Urban Complexes.

Key words: Migration, spatial practices, border and mobility.

\section{Introducción}

En Chile durante los últimos diez años se han observado cambios sustantivos en las migraciones y en la forma de abordar dichos estudios. Estos cambios están en concordancia con lo que ocurre a nivel mundial donde las migraciones muestran una aceleración de los flujos así como una dispersión entre lugares de origen y destino (OIM, 2015). El Departamento de Extranjería y Migración de Chile (Dem 2016) sostiene que Chile es un caso de interés en tanto se ha convertido en los últimos treinta años en destino de la migración fronteriza y transfronteriza de la región y en menor medida internacional ${ }^{1}$. Estos cambios obligan a una lectura teórica que transita desde una dimensión administrativa-nacionalista de la comprensión de la migración a una discusión centrada en las nociones teóricas que permitan comprender los proyectos migratorios, las temporalidades de los desplazamientos y el sentido que adquieren las prácticas transfronterizas.

En Chile nuevas corrientes de investigación prestan atención a aspectos menos explorados de la migración, en específico aquellas referidas a la aparición de circuitos migratorios y de prácticas fronterizas (Guizardi, Valdebenito, López y Nazal 2015; Lube y Garcés 2013a, 2013b; Lube, Heredia, Muñoz, Grecia y Valdebenito 2013; Lube, Penna, Vicuña y Pérez 2015; Tapia 2015) y la preocupación de la frontera y sus dispositivos de control como filtro para el ingreso (Liberona 2015a, 2015b); los motivos tras los cambios y trayectorias residenciales (Contreras y Palma 2015; Imilan, Garcés y Margarit 2014; Márquez 2013); al surgimiento de barrios o

\footnotetext{
* Proyecto FONDECYT No 1150123.

** Universidad de Chile, Facultad de Arquitectura y Urbanismo, Departamento de Geografía. Santiago, Chile. Correo electrónico: ycontrerasg@uchilefau.cl

*** Universidad Arturo Prat, Instituto de Estudios Internacionales INTE. Iquique, Chile. Correos electrónicos: marcela.tapia@unap.cl; nliberonac@gmail.com
} 
enclaves migrantes en Santiago y en regiones (Garcés 2012, 2015; Tapia y Chacón 2016), entre otros.

A partir del estudio de dos ciudades fronterizas, una ubicada al sur de Perú (Tacna) y la otra al norte de Chile (Arica), se analizan las prácticas socioespaciales semanales o mensuales de sus residentes. Se devela así el sentido de transfrontericidad de las prácticas y el afán de los habitantes de aprovechar las ventajas del cruce dando lugar a distintos tipos de movilidades. Por tanto, se asume una posición teórica y empírica que da sentido a los vínculos e interacciones que se producen entre Arica y Tacna, sorteando así el carácter geopolítico asignado a dos espacios de proximidad.

Tacna y Arica son capitales de sus unidades administrativas y tienen en común que están alejadas de los centros políticos de sus respectivos países. Ambas ciudades se encuentran ubicadas en la frontera chileno-peruana a una distancia de 58 $\mathrm{km}$. El interés por lo que ocurre en Arica y Tacna es doble. En primer lugar, el paso fronterizo de Chacalluta -el más septentrional del país- es el más cruzado a nivel nacional, registrando más de 40 millones de entradas y salidas entre 2000 y 2014. Se estima que las cifras rondan los 6 millones de cruces según datos obtenidos en la PDI y en el Complejo Fronterizo. Por último, Chacalluta es la principal puerta de entrada de los extranjeros que ingresan a Chile vía terrestre (Dem 2016, p. 46).

En segundo lugar, esta frontera tiene una carga histórica importante cuya delimitación impacta hasta hoy. Después de un largo litigio pos-Guerra del Pacífico que dejó a ambas ciudades en un limbo jurídico, se resolvió mediante el Tratado de 1929 que Arica quedaba para Chile y Tacna para Perú (González 2008). Si bien durante casi todo el siglo $\mathrm{XX}$ hubo controversias por esta frontera, estas se agudizaron a partir de finales de la centuria. Una serie de litigios, que no son objeto de este estudio, han tensionado la frontera durante las últimas décadas y el sentido que tiene ella tanto para sus residentes como para las autoridades nacionales.

Más allá de las controversias, desde la década de los años 90 se registra un importante movimiento de población entre ambas ciudades, lo que se expresa en un crecimiento del número de cruces de personas, automóviles y mercancías entre Arica y Tacna. Por tanto, se trata de una frontera de variadas interacciones que exigen discutir el sentido de las prácticas socioespaciales y la interpelación que estos movimientos hacen a las concepciones físicas $\mathrm{y}$ administrativas del término frontera.

El artículo se estructura en cuatro apartados. En el primer apartado se presenta una discusión teórica acerca del concepto frontera, regiones fronterizas y sus vínculos con las prácticas de movilidad de los cruzadores. En un segundo apartado se expone la metodología de investigación prestando atención a los resultados de la encuesta, así como también la construcción de las prácticas socioespaciales fronterizas. En la tercera parte se exponen los resultados de la encuesta, contextualizando las vocaciones y características socioespaciales de las dos urbes fronterizas, así como también los principales discursos que emergen de los entrevistados. La cuarta parte final está consagrada a develar nuevas preguntas, en tanto la dimensión desigualdad que reflejan las prácticas espaciales de dos espacios transfronterizos, exige repensar el sentido del término frontera.

\section{Revisión de la literatura. Las fronteras y las regiones transfronterizas}

El estudio de ciudades en frontera es reciente. En la actualidad se estima en 900 las ciudades con alguna connotación fronteriza y "unos 37 pares de ciudades que constituirían aglomeraciones urbanas de carácter binacional, lo que él llama 'conurbaciones transfronterizas"” (Dilla 2008, p. 17). En América Latina las ciudades y las regiones fronterizas fueron concebidas hasta fines del siglo XX como espacios marginales dentro de los Estados, solo recientemente se ha producido una mayor preocupación por dichos lugares (Núñez 2011). Durante el siglo XX las regiones extremas de Chile fueron vistas desde una perspectiva centralista, es decir, como zonas marginales y aisladas con una mayor preocupación por la línea política más que los espacios colindantes y sus habitantes (Núñez 2011). En los años 70 y 80 esta preocupación creció con la dictadura militar como orientación geopolítica y de defensa, lo que motivó la creación de una política de incentivo de ocupación de las regiones extremas y el minado de la frontera con Perú y Bolivia (Holahan 2006).

Más allá de Chile, los estudios de frontera han experimentado un importante auge desde los años 90 a la fecha. Newman (2006) refiere al "fin de las fronteras" tras la caída del Muro de Berlín en 1989. Los estudios fronterizos han transitado desde la definición de frontera como línea que separa o 
demarca a la preocupación de las fronteras como espacios de interacción y contacto entre habitantes (Zapata-Barrero y Ferrer-Gallardo 2012). "La investigación fronteriza ha dejado de ser el ejercicio analítico descriptivo que encarnó años atrás para convertirse en un fecundo y más heterogéneo campo de escrutinio de dinámicas socioespaciales" (Zapata-Barrero, R, 2012: 42).

En este contexto el debate actual de los estudios fronterizos (border studies) afirman que la frontera, como concepto y como categoría analítica, ha experimentado importantes cambios en sus significados y en sus funciones (Newman 2006). Respecto del significado existe un amplio desarrollo de esta noción en las Ciencias Sociales para señalar diferencias o demarcaciones entre grupos sociales, generaciones o grupos étnicos, por mencionar algunos (Kolossov 2005). En este sentido las fronteras han experimentado un cruce inter y transdisciplinario en la Ciencias Sociales (Newman 2015) para aludir casi siempre a diferencia o discontinuidad. Desde el punto de vista de las funciones, el análisis va más allá de la línea y en un sentido más versátil, porque ya no es solo instrumento de la política de los Estados y de control territorial, sino también actúa como "marcador de identidad y diferenciación social" (Paasi 2005, p. 666).

Las fronteras en tanto filtro (Heyman 2011) o cedazo (Liberona 2015a) que selecciona y permite o no el ingreso de las personas a un territorio experimenta cambios al incluirse nuevos dispositivos de poder y control. Dilla (2008) apela a la refuncionalización de las fronteras, ya que se redefinen sus roles en los procesos de reproducción capitalista. Dentro de la amplia producción acerca del término frontera interesa detenerse en aquella que se preocupa por la producción de espacios y regiones transfronterizas, es decir, áreas que tienen notables interacciones económicas y sociales y en muchos casos una historia compartida o un sustrato cultural común (Durand 2015).

Es posible distinguir al menos dos grupos de regiones fronterizas, aquellas que poseen vínculos institucionalizados especialmente por medio de la cooperación desde los Estados a los que pertenecen $\mathrm{y}$ otro formado por regiones o zonas que presentan interacción dada por vínculos formales, pero sobre todo informales que explican la continuidad y estabilidad de los lazos en el tiempo (Tapia, Liberona y Contreras 2017). Se asume por tanto la tesis de Morales (2010), para quien una región fronteriza "es una entidad que carece de estatuto jurídico en el ámbito de la geografía política, pero dentro de ella se hallan fuerzas sociales y relaciones de producción y de poder que funcionan en dependencia o con una cierta autonomía respecto de otras realidades" (Morales 2010, p. 187).

Lo que hace distinto a una región transfronteriza de otra es que estas tienen a la frontera como centro de referencia, por tanto es "un espacio de vida que traspasa las líneas de separación (límite) y origina una integración entre los territorios colindantes" (Morales 2010, pp. 189-190). Los estudios migratorios en regiones fronterizas han obligado a discutir las nociones utilizadas para referirnos a los desplazamientos en dichos lugares, especialmente el de migración (Heyman 2012). Diversos estudios en América Latina (Aponte 2012; Jaquet 2008; Parella 2014), Europa (Perkmann 2003; Zimmerbauer 2011) y Asia (Banerjee y Chen 2013; Hui, Wong, Li y Yu 2011), advierten de distintos tipos de movimientos de población en regiones, ciudades o conurbaciones fronterizas donde se identifican una variedad de desplazamientos que tienen en común la no instalación o establecimiento al otro lado de la frontera. Son cruces diarios, semanales o por días con fines laborales, comerciales, educacionales o sanitarios que buscan aprovechar la ventaja de pasar la frontera para luego retornar.

$\mathrm{Al}$ referir por tanto a la movilidad en regiones transfronterizas este estudio incorpora todos los movimientos cortos diarios, semanales o por días que no implican un cambio de residencia habitual como la movilidad laboral por temporadas o commuters en la frontera ${ }^{2}$. También se consideran todos los movimientos por compras, atenciones médicas o con motivos de ocio que no pueden ser considerados como migraciones. El tiempo, los motivos y la no instalación en el "otro lado", diferencian a este movimiento de las migraciones en sentido estricto y permiten captar algunos movimientos que ocurren en las regiones y ciudades fronterizas en distintas partes del mundo.

Los paradigmas de movilidad en la frontera advierten de los motivos del cruce, el sentido que las personas le asignan a los espacios de circularidad, la temporalidad de los movimientos y los vínculos que se establecen entre un espacio y otro. Es así como la movilidad con constructo socioespacial genera nuevas relaciones sociales y nuevas configuraciones de los contextos y marcos territoriales que alojan formas de vida social sustentadas en la 
movilidad. Tarrius (2007) "propone que algunas de estas movilidades no se merecen analizar como meros efectos de un nuevo tipo de sociedad sino que son productoras y estructurantes de formas de sociabilidad no localizadas" (Mallimaci 2012, p. 78) o mejor dicho, multisituadas. Por tanto, estos movimientos de las personas producen territorios circulatorios que son a su vez producto y condición de las movilidades.

\section{Descripción del área de estudio}

Arica y Tacna son ciudades fronterizas no conurbanas (Figura 1), estando separadas por un amplio desierto en casi 58 kilómetros. A nivel macro las dos ciudades presentan diferencias funcionales que se traducen en asimetrías y desigualdades en términos de sus economías, exigencias administrativas para realizar el cruce y políticas de desarrollo local. En el Cuadro 1 se presentan las diferencias entre la ciudad de Arica y Tacna, teniendo como limitantes que las fuentes consultadas son diferenciadas en año, pero al menos permiten tener una radiografía de los elementos que sustentan las diferencias en los flujos productivos y reproductivos de los encuestados. Las prácticas socioespaciales fronterizas productivas son aquellas vinculadas a la búsqueda de empleo y las reproductivas corresponden a aquellas relacionadas al ocio, recreación, medicina, entre otros.
Tanto Arica como Tacna son ciudades con menor jerarquía urbana y demográfica a nivel nacional, sin embargo, se configuran como espacios fronterizos de relevancia geopolítica. En el caso específico de Arica, esta se convierte en zona de resguardo del Estado-Nación durante la dictadura de Pinochet (1973 a 1987). Esa condición geopolítica le resta jerarquía económica y demográfica al menos entre los años 70 y 90. El 2016 Arica concentró casi el 1,2\% de la población chilena con aproximadamente 186 mil habitantes. Por su parte, Tacna es una ciudad intermedia peruana con casi 342 mil habitantes (INEI, 2016), cifra que representa el $1,1 \%$ del total país.

A nivel económico, los trabajadores de Arica se ocupan preferentemente en el comercio al por mayor y menor, repuestos, vehículos, automotores/ enseres domésticos (17,5\%); construcción (13,5\%); industrias manufactureras no metálicas $(10,9 \%)$, administración pública $(9,1)$, entre otras (Región de Arica y Parinacota 2016) ${ }^{3}$. La agricultura, ganadería, caza y silvicultura en conjunto tienen un $47 \%$ de la población en edad de trabajar ocupada en esas labores (Perfil Migratorio en Chile 2011) ${ }^{4}$. En cambio en Tacna, la principal economía se asocia a la extracción de minerales, petróleo y gas, seguido del comercio con una participación del $11,8 \%$; otros servicios no especificados con $15 \%$; agricultura y ganadería con $6,4 \%$ de participación

Cuadro 1. Características generales de las ciudades fronterizas de Tacna y Arica.

\begin{tabular}{|c|c|c|}
\hline Variable & Arica (Chile) & Tacna (Perú) \\
\hline Total de población & $157.568^{(1)}$ & $341.838^{(2)}$ \\
\hline Superficie* & $16.876 \mathrm{~km}^{2}$ & $16.075 \mathrm{~km}^{2}$ \\
\hline Densidad de población (hab/km²) (2014) & 11,2 & 18,0 \\
\hline Ingreso promedio per cápita & US\$279, $1^{(3)}$ & US\$ $242,6^{(7)}$ \\
\hline PIB per cápita (dólares) & US\$ $5063.4^{(4)}$ & US\$2431.3 $3^{(4)}$ \\
\hline Educación pública primera & 56 colegios $^{(4)}$ & 42 colegios $(4)$ \\
\hline $\mathrm{N}^{\circ}$ de hospitales (2016) & 1 & 2 \\
\hline Centros de salud & $21^{(4)}$ & $72^{(4)}$ \\
\hline Tasa de desocupación (\%) (5) & $9,7^{(5)}$ & $4,3^{(6)}$ \\
\hline
\end{tabular}

1 Proyecciones del INE, 2014.

2 INEI, Perú, 2016.

3 INE, 2011. En http://www.inearicayparinacota.cl/noticia.aspx ?opc=ShowNew\&id=158

4 En diario La Tercera, 2014. Datos obtenidos desde el sitio web http://www.latercera.com/noticia/politica/2014/02/674-564279-9-lasdiferencias-en-asignaciones-fiscales-que-tienen-chile-y-peru-con-sus.shtml

5 Encuesta CASEN, Encuesta de Caracterización Socioeconómica Nacional (CASEN), Ministerio de Desarrollo Social. 2009

6 Observatorio Socioeconómico Laboral (OSEL) de la Dirección Regional de Trabajo y Promoción del Empleo. En http://diariocorreo.pe/edicion/tacna/tacna-tasa-de-empleo-informal-es-de-72-9-584191/

7 INEI-ENAHO: 2007-2011.

* En el caso de Chile se consideró la superficie de la provincia de Arica y Parinacota y el caso de Perú del departamento de Tacna. 
(Caracterización del Departamento de Arequipa $2016)^{5}$. Sin embargo, la actividad extractiva no se configura en una fuente de empleo segura al menos para los encuestados en el paso fronterizo Santa Rosa-Chacalluta. Desde el 2000 el turismo se convierte en una fuente de ingreso significativa para la ciudad y la región donde se ancla.

El nivel de equipamientos expuestos en el Cuadro 1 exhibe desigualdades entre ambas ciudades. Tacna cuenta con dos hospitales de alcance local y regional: Hospital de la Solidaridad y Hospital Hipólito Salas, siendo el primero altamente demandado por los encuestados ariqueños que son parte del universo investigado. En la ciudad de Tacna existen al menos 72 centros de salud en comparación con los 21 existentes en Arica. Estas diferencias vuelven a Tacna un espacio representativo de la movilidad por salud transfronteriza.

Finalmente, respecto de los marcos normativos que regulan el cruce entre Tacna y Arica los últimos años han sido claves, ya que las autoridades migratorias regionales de los dos países permite a los ciudadanos peruanos ingresar con documento de identidad o pasaporte por el paso de Chacalluta en calidad de turista. El tiempo máximo que pueden permanecer en territorio chileno es de 90 días. Lo mismo se exige para los chilenos que viajan a Perú. Estas posibilidades del cruce vuelven a Tacna y Arica un espacio transfronterizo cuyas economías y modos de vida son complementarias y necesarias.

\section{Metodología}

La investigación es de carácter cuantitativo y cualitativo, sustentado en la aplicación de 240 encuestas en el paso fronterizo chileno de Chacalluta. La muestra se aplicó desde el 17 al 23 de julio del 2015. Como técnica de muestreo se ha utilizado el muestreo no probabilístico y de conveniencia a los objetivos y limitantes de una encuesta realizada en un cruce fronterizo. Así, fueron contempladas 54 preguntas orientadas a conocer las experiencias del cruce de residentes de Arica y Tacna; los motivos del cruce, la frecuencia y condiciones materiales y administrativas del cruce; las prácticas socioespaciales y los recorridos cotidianos de los encuestados así como también las limitantes al momento de transitar por el cruce Santa Rosa del lado peruano y Chacalluta del lado chileno.

Se escogieron diferentes franjas horarias de aplicación del instrumento (mañana, mediodía y noche), buscando así captar la mayor variedad de cruces y motivos de estos. El universo encuestado (240) estuvo compuesto por 147 peruanos, 91 chilenos, 1 ecuatoriano y 1 boliviana, de estos 121 son hombres y 119 mujeres, del total captamos 153 ingresos a Chile y 87 salidas del país.

Para profundizar la información obtenida en la encuesta, en el 2016 se llevó a cabo una segunda fase de trabajo de campo que incluyó entrevistas a informantes claves y cruzadores. Las entrevistas se realizaron en dos etapas entre julio y agosto, respectivamente. Para eso se diseñó una entrevista semiestructurada para informantes claves y un set de entrevistas por tipo de movilidad. Las entrevistas se aplicaron en Arica a distintos actores como Policía de Investigaciones (PDI), Municipalidad de Arica, Aduana, Terminal Internacional, Inspección del Trabajo, Paso Fronterizo de Chacalluta y Servicio Jesuita Migrante, entre otras. En Tacna se realizaron entrevistas en el Hospital de la Solidaridad, la Cámara de Comercio, restaurantes, Terminal Internacional de Tacna y Municipalidad de Tacna, entre otros informantes. Para efectos de la investigación se resguardan los nombres de los actores claves. La dimensión cualitativa se construyó a partir de una matriz de análisis que incluye tres dimensiones: discurso acerca de la frontera y el sentido de esta; el tipo de prácticas espaciales y los motivos del cruce; y finalmente, el recorrido desde el espacio de residencia hasta el lugar de destino, ya sea transitorio o permanente.

En la Figura 1 se localizan los espacios de residencias de los encuestados identificando así los lugares de residencia y las distancias cotidianas que recorren en función del tipo de prácticas espaciales que realizan. La espacialidad contempló dos tipos de motivos de cruce: en primer lugar aquellos vinculados a prácticas productivas, es decir, las estrategias orientadas a lograr trabajo, beneficios económicos o medios materiales de subsistencia (Parella, 2013), en segundo lugar se analizaron aquellas que comprenden acciones que aseguran la "reproducción social, vivienda, acceso a servicios sociales (educación, salud y saneamiento, seguridad) y recreación" (Morales 2010, p. 191). Las cartografías construidas tomaron como base las direcciones y lugares declarados en el levantamiento.

Se construyó una cartografía de las prácticas espaciales de los entrevistados según lugar de residencia o salida (origen, color morado en la Figura 1) y lugar de destino. El proceso de georreferenciación se realizó mediante los motores de 


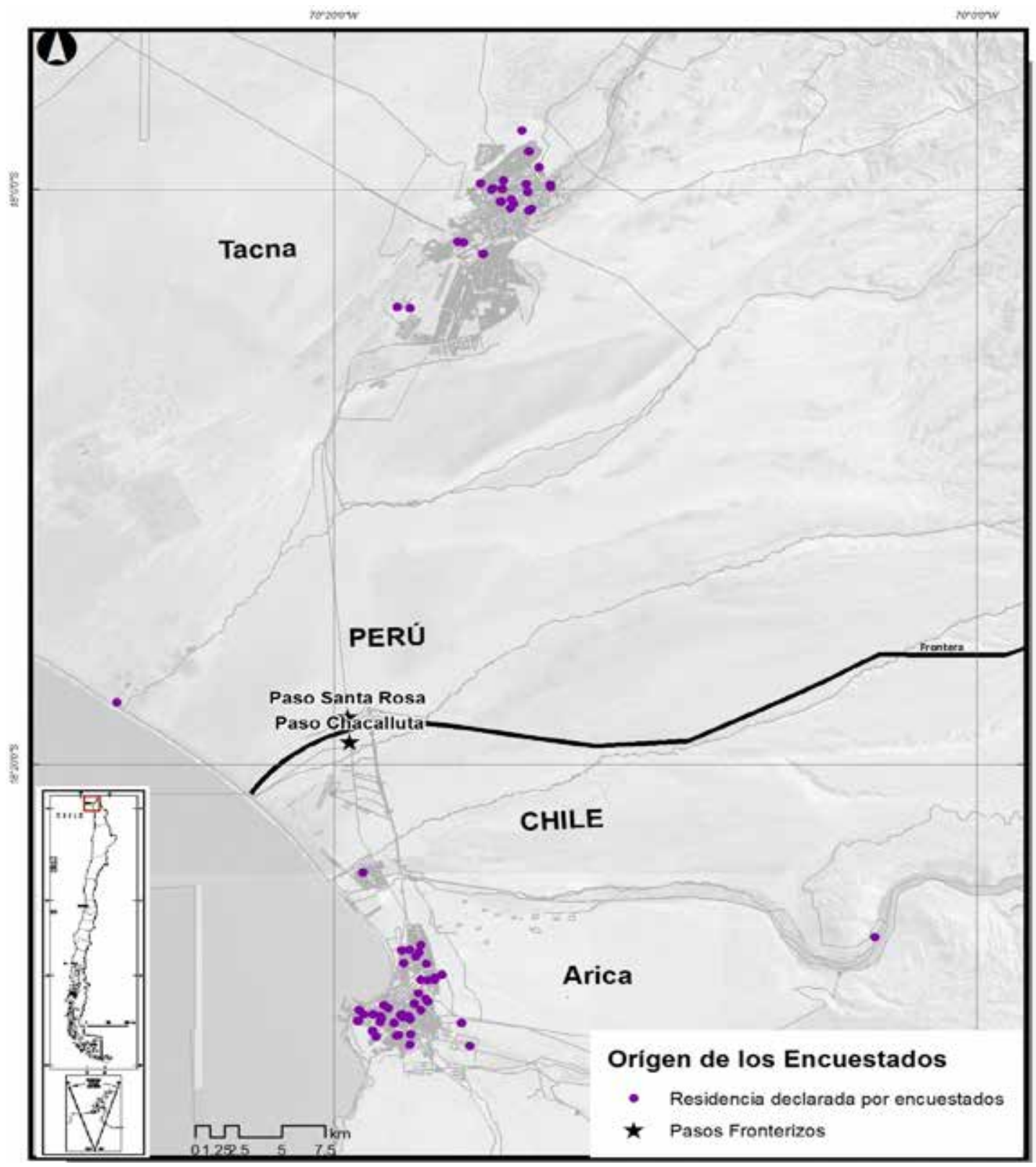

Figura 1. Espacios de análisis de dos ciudades fronterizas Tacna y Arica y lugar de residencial de los encuestados. Fuente. Autoras a partir de google earth, 2016 y encuestas, julio 2015.

búsqueda de Google earth para ambas ciudades, dando como resultado dos coberturas de geometría puntual expuestas en la Figura 1: con las localizaciones declaradas de origen y destinos. El identificador común que permitió la georreferenciación fue el número de encuesta. Mediante Sistemas de Información Geográfica SIG-ArcGis se espacializó la recta euclidiana que unificó las localizaciones de origen y destino, creando una nueva cobertura de geometría lineal que permitiese representar el sentido de las prácticas productivas y reproductivas de los 240 encuestados. En las tres coberturas de trabajo se les asoció la base de datos y para los casos de origen y destino se les creó una respectiva cobertura que contuviera el resumen de datos. Con esta metodología se puede 
determinar la distancia promedio recorrida entre los encuestados, determinar los barrios de origen, las concentraciones de destinos dentro de la ciudad y la comparación con características de los entornos y el tipo de prácticas espaciales, tanto productivas como reproductivas.

\section{Análisis de los resultados}

\section{Movilidad y prácticas productivas y reproductivas en el espacio transfronterizo de Arica-Tacna}

Los flujos productivos están representados en la Figura 2 y los reproductivos en la Figura 3. Cada punto del plano representa un destino. Las fechas en escala de grises grafican el recorrido de la práctica espacial. El tamaño del círculo refiere a la cantidad de cruzadores según este tipo de prácticas, sean productivas o reproductivas.

Del análisis de la encuesta y la espacialización de estas, se observa que los mayores flujos de movilidad son desde Tacna hacia Arica. 147 encuestados declararon cruzar hacia Arica por prácticas productivas (Figura 2) asociadas al trabajo $(36,1 \%)$. El 29,3\% de las personas, en cambio, se mueve por prácticas reproductivas, así como se evidencia en la Figura 3. Estos últimos flujos están orientados a los intercambios comerciales, la compra de productos en Arica o bien la comercialización de ellos. Otros casos declararon en $20,4 \%$ que los motivos del cruce estaban vinculados a asuntos personales, lo que permite plantear la tesis de la presencia de sujetos sociales que buscan trabajo por primera vez por las redes sociales preexistentes en Arica. En esta línea, algunos de los entrevistados de nacionalidad peruana y que trabajan en Arica plantean la necesidad de tener un contacto antes de venir a trabajar a Chile, aunque también existe la posibilidad de comprar los nexos por medio de una agencia de empleos, los que no necesariamente son formales, pero permitirían acceder a mejores fuentes de trabajo.

La recursividad de las prácticas productivas entre Arica y Tacna tienden a ser históricas. El carácter cíclico de ellas está estrechamente vinculado a las situaciones económico-políticas del Estado-Nación respectivo.

"Siempre fue atractivo Arica, somos de frontera y en una época, la época de Salvador Allende que es por el problema económico que tuvieron ustedes que lo que era Arica nos convenía mucho ir, yo estaba en la universidad en esa época el año 73. Había muchas cosas que se compraban en Arica a precios cómodos y era un comercio cómo se llamaba antes de pacotilla (acarreo de mercaderías a través de la frontera)" (hombre, dentista, 75 años, tacneño).

\begin{abstract}
"Siempre hemos tenido períodos de bonanza en uno y otro lugar... los vínculos con Tacna eran de ese tipo, mientras hayan condiciones mejores para atender en Arica, se atiende en Arica" (hombre, 60 años, funcionario del Terminal Internacional de buses, residente en Arica).
\end{abstract}

Asimismo, los cruzadores encuestados y entrevistados coinciden en que el ciclo entre los dos países es variable y que los hechos a nivel nacional se expresan en disparidades en las ciudades de Arica y Tacna, lo que implica las condiciones de ventaja/ desventaja en términos laborales, económicos y comerciales en una y otra ciudad. Estas medidas se manifiestan en diferencias en tipo de cambio y también producto del auge económico de Tacna y rezago estatal-regional de la ciudad de Arica.

Las Figuras 4 y 5 advierten además que los rangos promedios recorridos según prácticas productivas y reproductivas son similares. Sin embargo, en las prácticas productivas hay una mayor distancia desde Tacna a Arica, principalmente por las distancias de origen de los encuestados que pertenecen al sector norte de la ciudad peruana y posiblemente por las dificultades de movilidad. La mayoría de los residentes en Tacna provienen de barrios y pueblos jóvenes de bajos ingresos, especialmente desde el sector industrial Ciudad Nueva. Algunos encuestados habitan en sectores como Gregorio Albarracín y Cono Sur (Figura 4).

Las prácticas espaciales productivas desde Tacna hacia Arica y expuestas en la Figura 4 presentan un patrón espacial disperso en torno a los valles de Azapa y Lluta, los mercados de abastos (ASOAGRO), y las diferentes ferias libres localizadas en el centro de Arica como en espacios periféricos residenciales. En las entrevistas se identifica a los valles de Azapa y Lluta como espacios concentradores de población peruana en Arica, dedicándose especialmente a trabajos agrícolas en un escenario generalmente de desregulación de la fuerza laboral (Rojas y Vicuña 


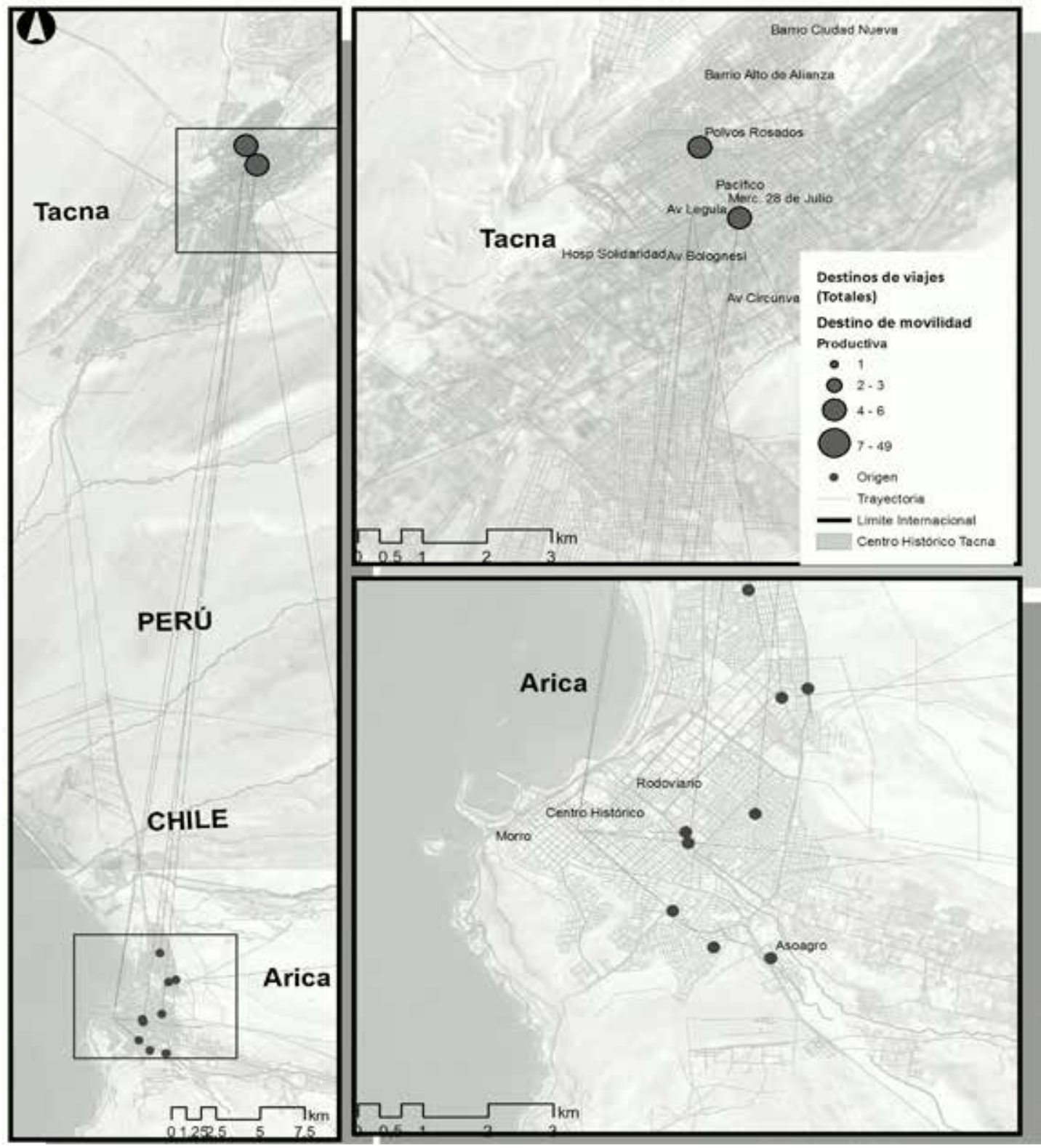

Figura 2. Distancias recorridas según prácticas espaciales cuyo origen es Tacna. Fuente. Elaboración propia a partir de Encuesta de Movilidad, julio 2015.

2014). Estos espacios se constituyen como los invisibilizados en las estadísticas pero visibilizados en los discursos de los entrevistados.

"El flujo que tiene relación con los peruanos está muy asentado en los que es el terminal, y en el sector del Valle de Azapa del kilómetro 30 hacia arriba que es el sector del Rápido, donde es otro flujo, que bajo el mismo contexto, buscan empleo, bajan de los sectores del valle entre las 5 y 6 de la mañana para poder establecerse en un lugar que es el sector que es pan de azúcar, para que un empleador pueda pasar por ellos, tanto varones como mujeres..., trabajan como maestro de construcción o temporero agrícola, y las mujeres como asesoras de hogar o garzona, el tema de la residencia es 


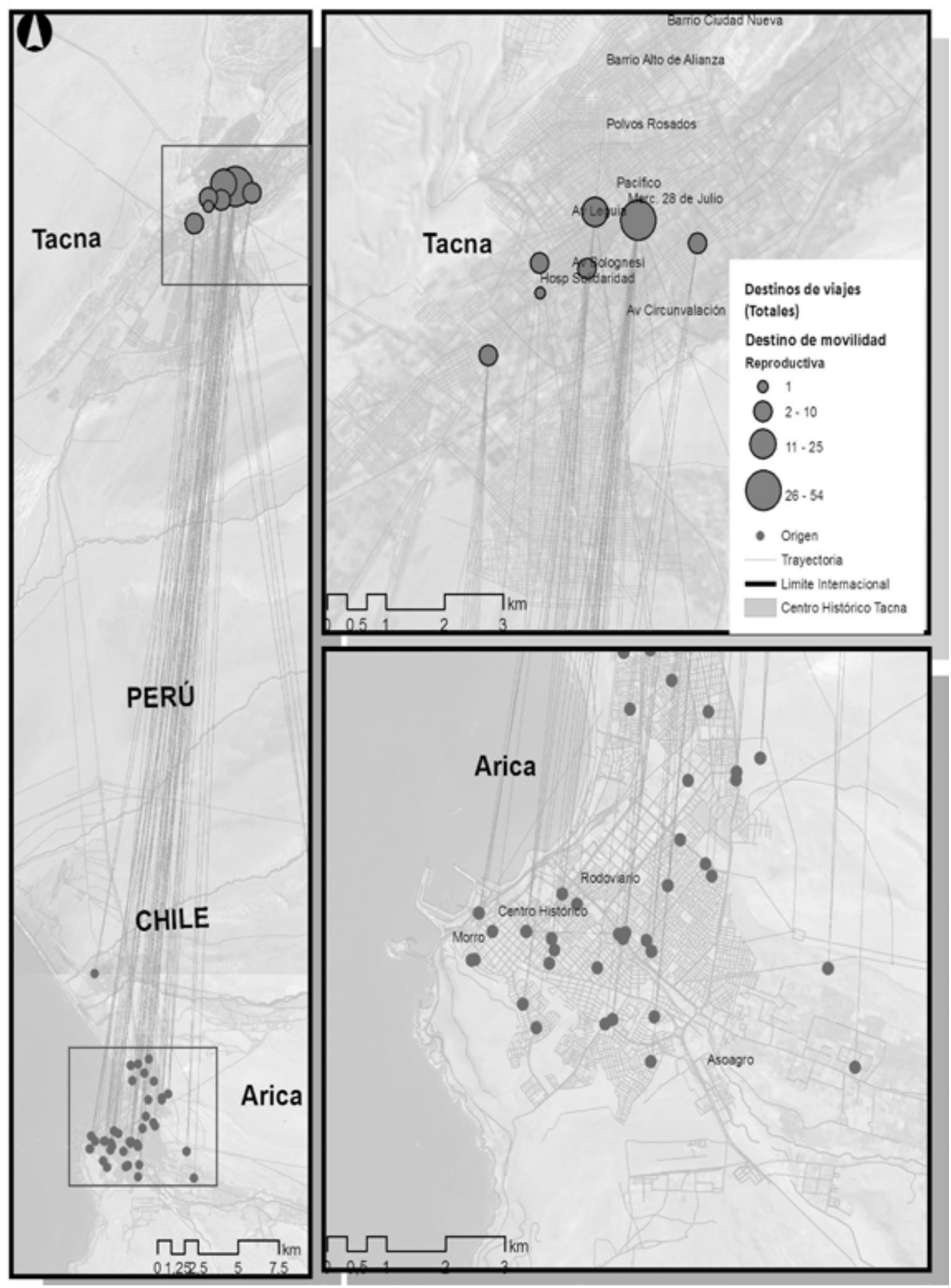

Figura 3. Las prácticas reproductivas prácticamente tienen distancias muy similares entre ambas rutas. Fuente. Elaboración propia a partir de Encuesta de Movilidad, julio 2015. 


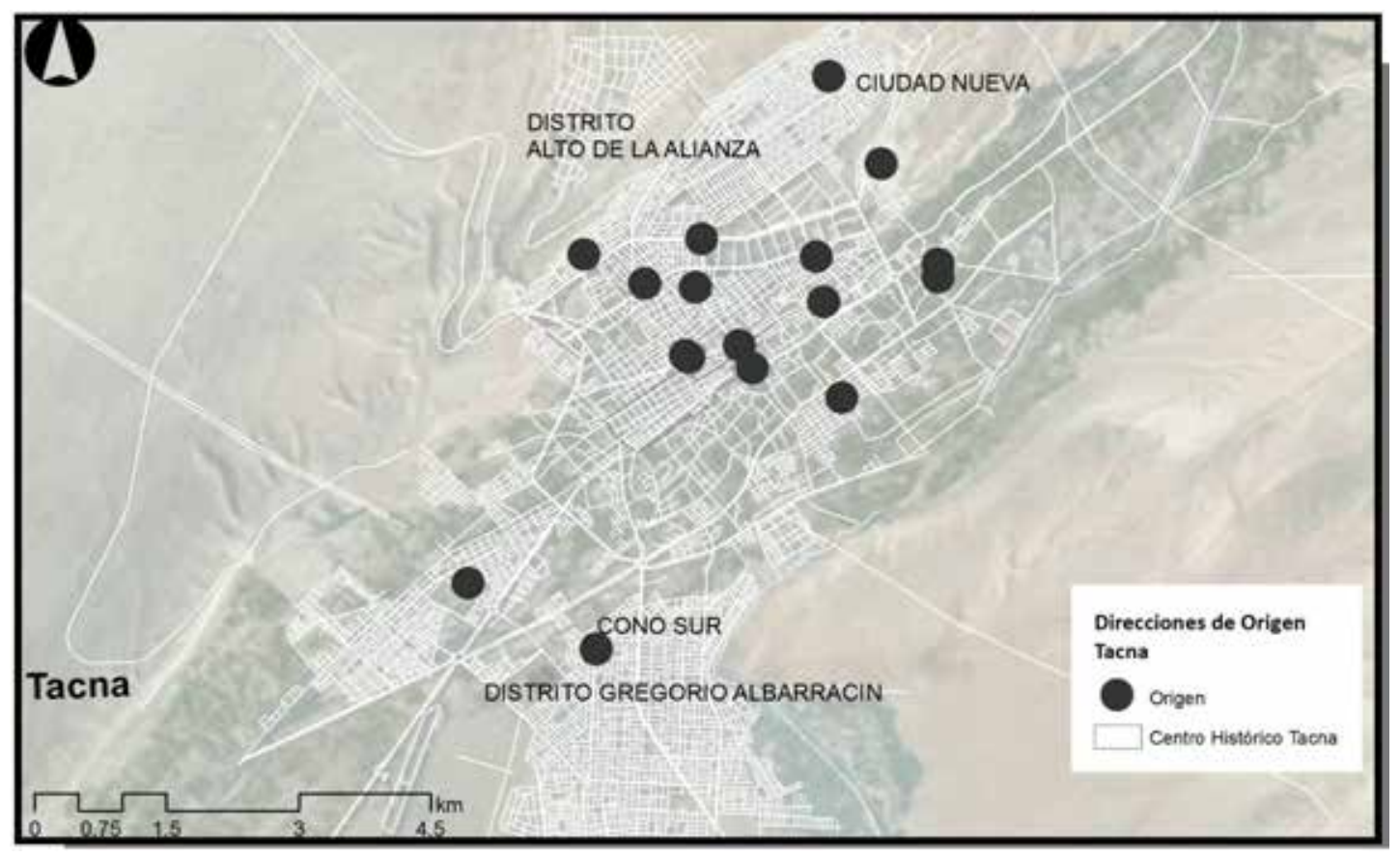

Figura 4. Distancias recorridas según prácticas espaciales cuyo origen es Tacna Fuente. Elaboración propia a partir de Encuesta de Movilidad, julio 2015.

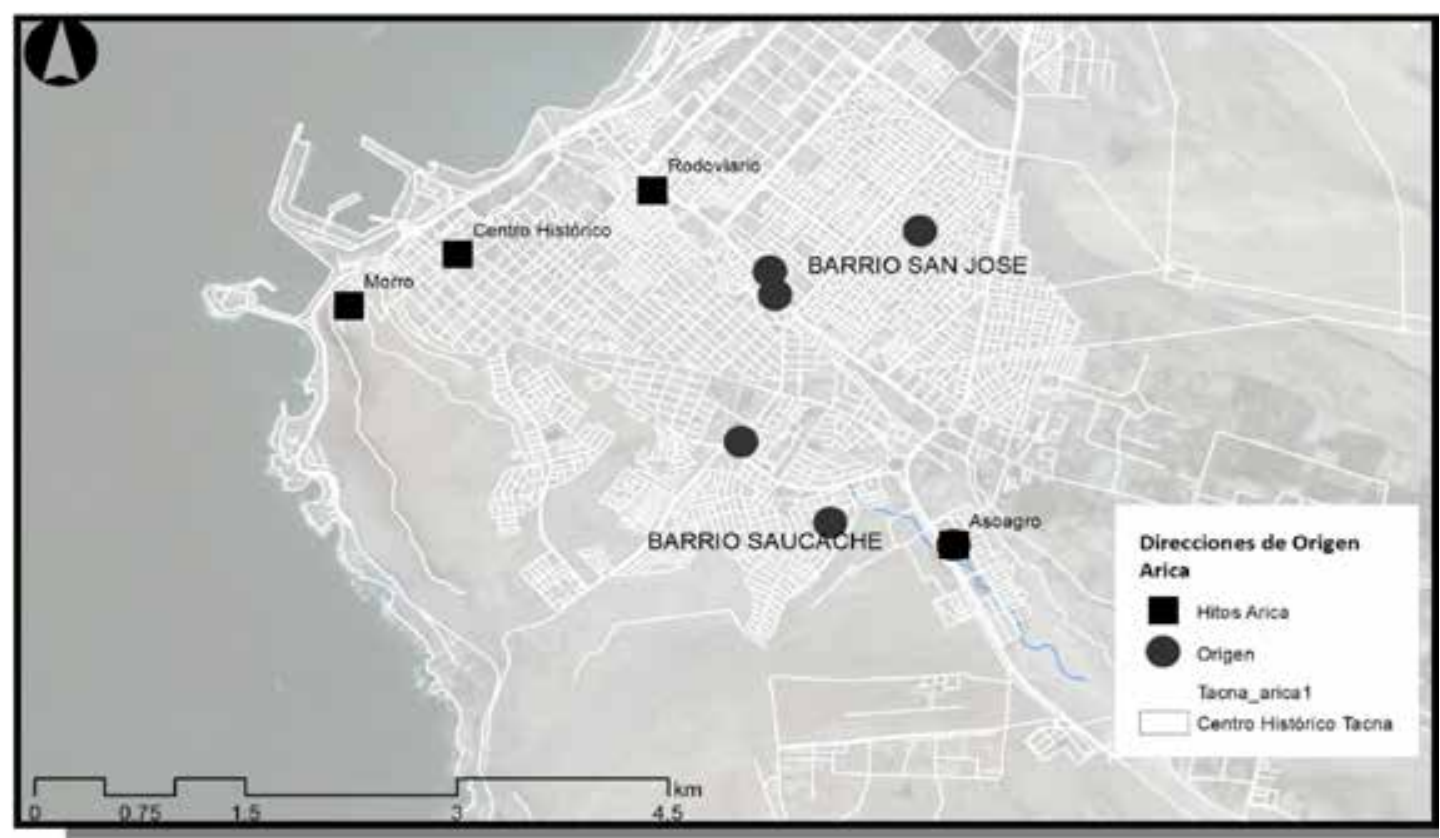

Figura 5. Las prácticas reproductivas prácticamente tienen distancias muy similares entre ambas rutas Fuente. Elaboración propia a partir de Encuesta de Movilidad, julio 2015. 
que como son trabajos temporales pueden ganar entre 10.000 y 12.000 pesos, no más que eso" (Trabajadora Social, Servicio Jesuita a Migrante, residente en Arica).

Lo anterior coincide con lo que Sassen (2007) define como la expansión de la economía informal, en tanto reductora de los costos de producción, y como mecanismo no exclusivo a territorios fronterizos. Este tipo de prácticas favorece la flexibilización y desregulación de la fuerza de trabajo.

Por otro lado, las prácticas reproductivas evidencian la centralidad de Avenida Bolognessi en la ciudad de Tacna. Este eje aglutina diferentes comercios vinculados a la venta de ropa, así como también los flujos comerciales de ariqueños que frecuentan el centro histórico, el Mercado de la Caplina, Plaza Veas, las ferias Polvos Rosados, 28 de Julio y la Galería Solari, entre otras. Los comercios que complementan la movilidad por salud también son significativos. El Hospital de la Solidaridad
(Figura 6) se configura en un ícono de la movilidad por salud hacia Tacna. Este centro asistencial fue creado con fondos del Municipio de Lima en el 2009, que alberga más de 60 especialidades médicas ubicadas en un único espacio, con costos de atención más económicos respecto de los centros médicos de Arica y Chile. En torno al Hospital se han instalado algunos servicios de exámenes médicos y farmacias que se vinculan a la Avenida Bolognesi donde se ubican centros oftalmológicos, farmacias, centros dentales, entre otras economías de aglomeración funcionales al Hospital. Las necesidades médicas por especialidades como oftalmología o dentista concentran la mayor atracción para los chilenos debido a los bajos precios y la rapidez del servicio, siendo un elemento de la historia reciente (70 en adelante) y que ha permitido dinamizar este rubro de la economía en la ciudad de Tacna.

"El hospital de la Solidaridad es un hospital de la municipalidad de Lima y la

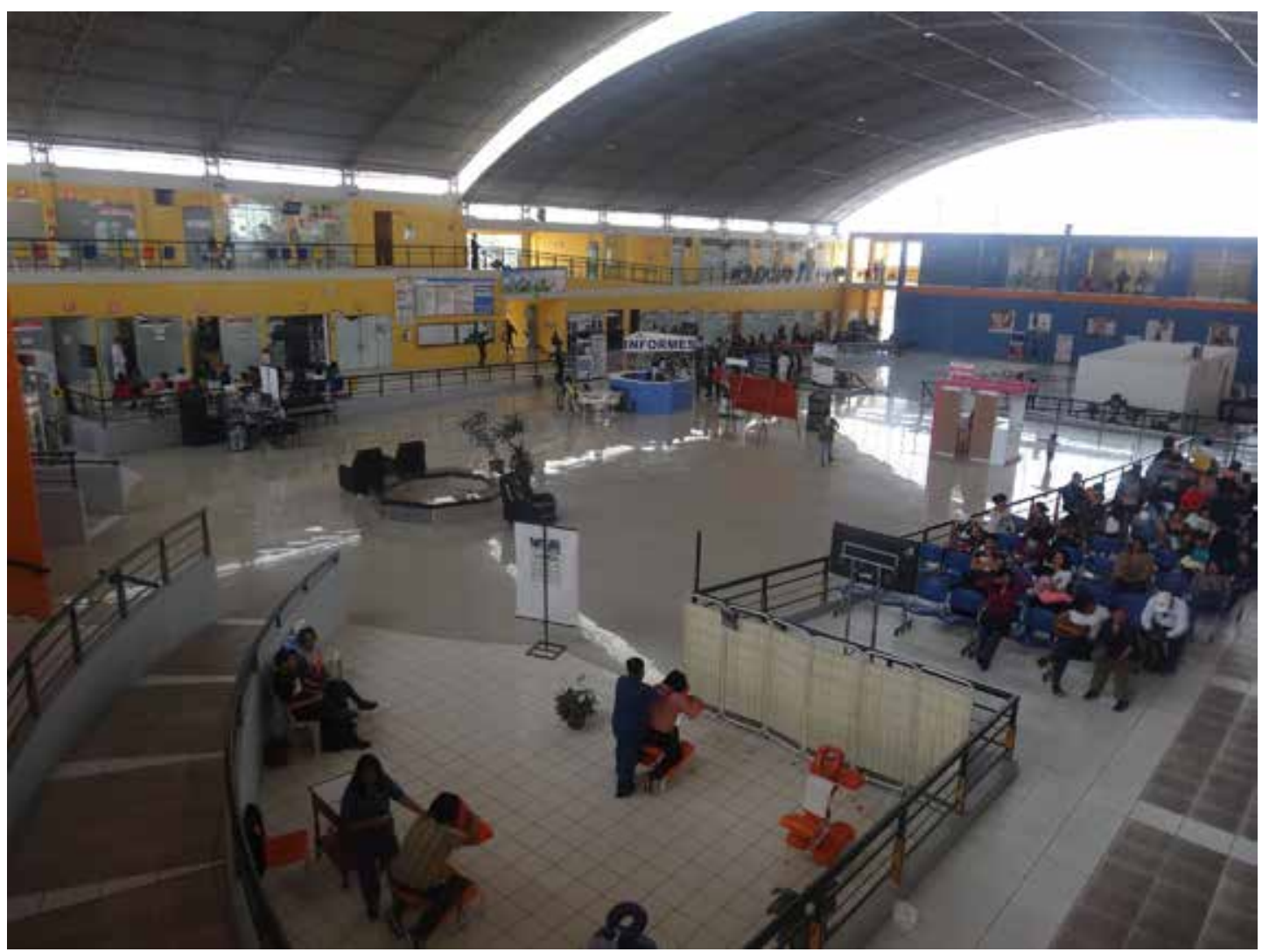

Figura 6. Hospital de la Solidaridad.

Fuente: autoras, septiembre 2015. 
municipalidad de Lima lo tiene en Tacna, lo puso como un hospital para los pobres originalmente para los pobres de Tacna, pero terminó siendo un hospital para los chilenos... le gusta la gente chilena que es la rapidez, porque lo ven rápido sus análisis, es un factor tienen un especialista y en Tacna hay especialistas" (hombre, dentista, 75 años, tacneño).

Esta situación deja la percepción de que la ciudad de Tacna se orienta a la atención del público chileno y a una idea bastante extendida de Tacna no vive si no es por los chilenos. Asimismo, Tacna se configura como una ciudad más económica para los jubilados chilenos y que es posible que se convierta en un destino de la migración de pensionados como ocurre en España:

\begin{abstract}
"Mito urbano ya cada vez suena más el rumor de que hay muchas personas que están yendo a vivir su vida de jubilados a Tacna, como no cuesta mucho cruzar la frontera, donde puedes venir a cobrar su pensión y allá les cunde más, arriendan y viven tranquilos y también trabajadores mineros que están permaneciendo en Tacna con sus familias, algo que circula en los últimos tiempos" (mujer, 60 años, funcionaria, Dirección del Trabajo, residente en Arica).
\end{abstract}

El análisis de las encuestas y la espacialidad de estas permite comprender que Arica-Tacna constituye lo que Dilla (2015) denominó como Complejo Urbano Transfronferizo (CUT), en tanto territorialidad que permite complementariedad, indistintamente la situación geopolítica de las capitales nacionales. Aún más, la proximidad es un recurso y estrategia a la que recurren quienes viven en espacios de frontera. Gran parte de los entrevistados manifiestan que los litigios fronterizos, desde una óptica nacional, no afectan el sentido, la temporalidad ni la recursividad de los movimientos entre territorios. Las fronteras se desdibujan a partir de la cotidianidad y complementariedad en las prácticas productivas y reproductivas.

\section{Conclusión}

A partir del estudio realizado postulamos que las prácticas socioespaciales fronterizas que despliegan los habitantes de Arica y Tacna en sus constantes movilidades productivas y reproductivas son la base de la formación de lo que Dilla llamó Complejos Urbanos Transfronterizos. Ambas ciudades están próximas y pese los trámites administrativos para cruzar el paso fronterizo las diferencias y desigualdades expresadas en la frontera y en su definición como demarcador hacen que Arica y Tacna funcionen como espacios diferenciados y contradictorios, pero complementarios. Tanto las dinámicas económicas oscilantes en conformidad con las políticas de cada país, las brechas sociales y económicas y los planes de desarrollo de ambos municipios favorecen esta desigualdad que explican la creación de espacios interdependientes en términos de mercado laboral y de consumo. Esta polarización socioeconómica redunda en la generación de una demanda de trabajadores informales infrapagados en Arica, la constitución de mercado laboral y comercial fronterizo de pequeña escala en ambos lados de la frontera y el surgimiento de un mercado de consumo y de salud que tiene por principal destinatario a los habitantes de Arica, pero no exclusivamente.

El paso fronterizo de Chacalluta (Chile)-Santa Rosa (Perú) es una frontera que tiene menos de un siglo (1929), en donde las históricas ciudades de Arica y Tacna se vinculan e interaccionan gracias a las prácticas socioespaciales productivas y reproductivas que expresan a su vez en tipos de movilidades. Así, las movilidades fronterizas se vuelven cotidianas en este espacio en que la suma de desigualdades internas de las ciudades con el resto de sus respectivos países va generando una interdependencia interurbana en el que cada ciudad depende de la otra para su desarrollo.

Sin embargo, el aumento del flujo entre ambas ciudades y un claro sentido de necesidad mutua no implica que desaparezca el sentido de frontera. Más bien, las fronteras siguen siendo el espejo de la consolidación de estados nacionales que establecen un interior y exterior y el recordatorio de un conflicto decimonónico. De hecho, no desaparece el nacionalismo y de cuando en cuando aflora en los momentos en que las efemérides recuerdan la configuración de la línea o por litigios limítrofes, pero estos no son suficientemente poderosos para detener el flujo entre ambas ciudades. La percepción de necesidad mutua hace que las tensiones diplomáticas se vivan en ambas ciudades con mayor independencia de lo que ocurre en las cancillerías o en las capitales respectivas. $\mathrm{La}$ frontera no desaparece ni se desdibuja, pero sí hay 
una clara necesidad de acortar las distancias entre ambas ciudades y favorecer el cruce. De hecho, una aspiración de las autoridades fronterizas es materializar el control integrado en la frontera para disminuir los tiempos del cruce.

La inclusión del estudio de las prácticas socioespaciales productivas y reproductivas y su expresión en tipos de movilidades (laboral, comercial, turismo, ocio, salud, gastronómico, entre otros) permiten identificar las complementariedades entre ambas ciudades. Estas prácticas son las que permiten observar las movilidades que configuran a las ciudades fronterizas en espacios comunes, interdependientes y transfronterizos. La concentración de las prácticas productivas desde Tacna hacia Arica devela a esta ciudad como destino laboral, mayoritariamente informal, constituyendo a la ciudad y los valles en un mercado laboral fronterizo. Desde las prácticas reproductivas se advierte un patrón de concentración y una economía de aglomeración de comercios, turismo y centros médicos en la ciudad de Tacna, que se localizan en torno a la Avenida Bolognesi y centro de Tacna. Por tanto, es necesario insistir y potenciar esa ausencia de un centro de gravedad en tanto ambas ciudades fronterizas se necesitan como aliadas, más que como competidoras. Los ariqueños y tacneños saben que la frontera entre ambos países es una línea de la concordia entre sus deseos de crecer, mejor y en paz.

El estudio de las ciudades fronterizas aporta a una mayor comprensión de la movilidad en frontera y a los estudios migratorios en general. Hasta ahora y debido al centralismo del país la comprensión del fenómeno migratorio latinoamericano se ha especializado en comprender las transformaciones que los inmigrantes producen en las ciudades y en la sociedad receptora. Sin embargo, la atención por las regiones fronterizas ha permitido tensionar los conceptos de los estudios migratorios y aportar nuevas nociones para comprender lo que ocurre en las ciudades fronterizas. Por tanto, los espacios fronterizos no se configuran como sitios administrativos, más bien son reflejo de espacios y lugares en constante resignificación de los capitales y recursos de movilidad, porque los sujetos sociales van creando una espacialidad entre una ciudad y otra indistintamente la temporalidad de ese recorrido. Esto último exige comprender las fronteras como espacios ni absolutos ni naturales, más bien como espacios contradictorios donde la interacción es vital para el desarrollo de las ciudades gracias y a pesar de la existencia de la línea.

\section{Referencias Citadas}

Aponte, J.

2012 Comercio y ocio en la transformación del espacio urbano fronterizo de Leiticia y Tabatinga. In C. Zárate, J. Pierre, C. Leonor, J. Picón, O. Thomas, R. Steiman, A. Berno, J. C. Peñan, J. Aponte, C. Toledo y N. Victorino (Eds.), Espacios urbanos y sociedades transfronterizas en la Amazonía (pp. 205-235). Leticia: Universidad Nacional de Colombia.

Banerjee, P. y Chen, X.

2013 Living in in-between spaces: A structure-agency analysis of the India-China and India-Bangladesh borderlands. Cities, $34,18-29$.

Cabrera, N.; Jugo, L. y Reátegui, J.

2016 Caracterización del Departamento de Arequipa. Banco Central de Reserva del Perú. Arequipa, Perú.

Castillo, M. y Nájera, J.

2014 México como país de origen, tránsito y destino de migrantes, una revisión a partir de la emif norte y la emif sur. In Consejo Nacional de Población (Ed.), 20 años de la Encuesta sobre Migración en la Frontera Norte de México (pp. 17-35).

Contreras, Y. y Palma, P.

2015 Migración latinoamericana en el área central de Iquique: Nuevos frentes de localización residencial y formas desiguales de acceso a la vivienda. Anales de Geografía, 35 (2), 45-64
Chávez, S.

2016 Border Lives: Fronterizos, Transnational Migrants, and Commuters in Tijuana. New York: Oxford University Press.

DEM

2016 Migración en Chile 2005-2014. Santiago de Chile: Sección Estudios del Departamento de Extranjería y Migración, Ministerio del Interior.

Dilla, H.

2008 Las ciudades en las fronteras: introducción a un debate. In H. Dilla (Ed.), Ciudades en las fronteras. Aproximaciones críticas a los complejos urbanos transfronterizos (pp. 1729). Santo Domingo: Editora Manatí.

Durand, F.

2015 Theoretical Framework of the Cross-border Space Production-The Case of the Eurometropolis LilleKortrijk-Tournai. Journal of Borderlands Studies, 30(3), 309-328.

Garcés, A.

2012 Localizaciones para una espacialidad: territorios de la migración peruana en Santiago de Chile. Chungara, Revista de Antropología Chilena, 44 (1), 163-175.

Garcés, A.

2015 Migración peruana en Santiago. "Prácticas, espacios y economías”. Santiago de Chile: RIL Editores. 
González, S.

2008 La llave y el candado: el conflicto entre Perú y Chile por Tacna y Arica (1883-1929). Santiago de Chile: LOM.

Guizardi, M.; Valdebenito, F.; López, E. y Nazal, E.

2015 Condensaciones en el espacio hiperfronterizo: apropiaciones migrantes en la frontera norte de Chile. In M. Guizardi (Ed.), Las fronteras del transnacionalismo. Límites $y$ desbordes de la experiencia migrante en el centro y norte de Chile (pp. 224-257). Santiago de Chile: Ocholibros.

Heyman, J.

2011 Cuatro temas en los estudios de la frontera contemporánea. In N. Ribas (Ed.), El Río Bravo Mediterráneo. Las regiones fronterizas en la época de la globalización (pp. 81-97). Barcelona: Bellaterra.

Heyman, J.

2012 Construcción y uso de tipologías: movilidad geográfica en la frontera México-Estados Unidos. In M. Ariza y L. Velasco (Eds.), Métodos cualitativos y su aplicación empírica. Por los caminos de la investigación sobre migración internacional (pp. 419-454). México D.F: Instituto de Investigaciones Sociales-UNAM/El Colegio de la Frontera Norte.

Holahan, D.

2006 El uso de minas terrestres en Chile: hacia una teoría de la frontera militar. Civitas-Revista de Ciências Sociais, 5(2), 343-351.

Hui, E.C.M.; Wong, F.K.W.; Li, S.M. y Yu, K.H.

2011 Integrations, identity and conflicts: A cross-border perspective on residential relocation of Hong Kong citizens to Mainland China. Habitat International, 35(1), 74-83.

Imilan, W.; Garcés, A. y Margarit, D.

2014 Poblaciones en movimiento. Etnificación de la ciudad, redes e integración. Santiago de Chile: Ediciones Universidad Alberto Hurtado.

Jaquet, $\mathrm{H}$.

2008 Más allá de la frontera, las fronteras: una aproximación socioespacial a las situaciones fronterizas de Misiones, Argentina. In H. Dilla Alfonso (Ed.), Ciudades en la frontera. Aproximaciones críticas a los complejos urbanos transfronterizos (pp. 33-66). Santo Domingo.

Kolossov, V.

2005 Border Studies: Changing Perspectives and Theoretical Approaches. Geopolitics, 10(4), 606-632.

Liberona, $\mathrm{N}$

2015a La frontera cedazo y el desierto como aliado. Prácticas institucionales racistas en el ingreso a Chile. Polis. Revista Latinoamericana, 42, En: https://polis.revues. org/11308\#quotation

Liberona, $\mathrm{N}$.

2015b La rigidez de las fronteras. Inmigración e integración en Tarapacá (1990-2007). In M. Lube y A. Grimson (Eds.), Las fronteras transnacionales del transnacionalismo: límites $y$ desbordes de la experiencia migrante en el centro y norte de Chile (en prensa). Santiago de Chile: Ocho Libros.

Lube, M. y Garcés, A.

2013a Circuitos migrantes. Itinerarios y formación de redes migratorias entre Perú, Bolivia, Chile y Argentina en el norte grande chileno. Papeles de Población, 19 (78), 65-110.

Lube, M. y Garcés, A.

2013b Recortes y representaciones espaciales en los estudios sobre la migración peruana en Chile. La invisibilización del Norte Grande como locus de investigación. Anales.
Lube, M.; Heredia, O.; Muñoz, A.; Grecia, R. y Valdebenito, F. 2013 Experiencia migrante y apropiaciones espaciales: una etnografía visual en las inmediaciones del Terminal Internacional de Arica (Chile). Revista de Estudios Sociales, 48, 166-175.

Lube, M.; Penna, C.; Vicuña, J.T. y Pérez, C.

2015 Claves conceptuales e históricas para comprender la frontera norte de Chile y la migración en Arica y Parinacota. In J. T. Vicuña y T. Rojas (Eds.), Migración internacional en Aroca y Parinacota: Panoramas y tendencias de una región fronteriza (pp. 19-36). Santiago de Chile: Servicio Jesuita Migrante.

Mallimaci, A.I.

2012 Movilidades y permanencias. Repensando la figura del movimiento en las migraciones. Revista de Temas de Antropología y Migración, 77-92.

Márquez, F.

2013 De territorios, fronteras e inmigrantes: representaciones translocales en La Chimba, Santiago de Chile. Chungara (Arica), 45(2), 321-332.

Morales, A.

2010 Desentrañando fronteras y sus movimientos transnacionales entre pequeños Estados. Una aproximación desde la frontera Nicaragua-Costa Rica. In M. E. Anguiano y A. M. López (Eds.), Migraciones y frontera. Nuevos contornos para la movilidad internacional (pp. 185-224). Barcelona: Icaria.

Newman, D.

2015 Revisiting good fences and neighbours in a postmodern world after twenty years: theoretical reflections on the state of contemporary border studies. Nordia Geographical Publications, 44 (4), 13-19.

Núñez, A.

2011 Territorios fronterizos, territorios aislados: conceptos dinámicos de construcción histórica (procesos de significación). In F. Arenas, A. Salazar y A. Núñez (Eds.), El aislamiento geográfico: ¿Problema u oportunidad? (pp. 140-157). Santiago de Chile: Instituto de geografía, Pontificia Universidad Católica.

Paasi, A.

2005 Generations and the 'Development' of Border Studies. [Article]. Geopolitics, 10(4), 663-671.

Parella, S.

2014 Una exploración de las prácticas transfronterizas en la zona urbana Caléxico (Estados Unidos)-Mexicali (México). In M. Tapia y A. González (Eds.), Regiones fronterizas, migración y los desafios para los Estados nacionales latinoamericanos (pp. 41-69). Santiago de Chile: RIL Editores.

Perfil Migratorio en Chile

2011 Informe elaborado para la Organización Internacional para las Migraciones por Carolina Stefoni. Buenos Aires, Argentina.

Perkmann, M.

2003 Cross-Border Regions in Europe: Significance and Drivers of Regional Cross-Border Co-Operation. European Urban and Regional Studies, 10(2), 153-171. En: http://eur. sagepub.com/content/10/2/153.abstract

Región de Arica y Parinacota

2016 Instituto Nacional de Estadísticas. Dirección Regional Arica y Parinacota. http://www.inearicayparinacota.cl/ 
Rojas, N. y Silva, C.

2016 La migración en Chile: breve reporte y caracterización. Madrid: OBIMID Observatorio Iberoamericano sobre movilidad humana, migraciones y desarrollo.

Rojas, N. y Vicuña, J.T.

2014 Migración y Trabajo. Estudio y propuestas para la inclusión sociolaboral de migrantes en Arica. Santiago de Chile: Ciudadano Global/OIM.

Sassen, S.

2007 Una sociología de la globalización. Buenos Aires: Kast.

Tapia, M.

2015 Frontera, movilidad y circulación reciente de peruanos y bolivianos en el norte de Chile. Estudios Atacameños. Arqueología y Antropología Surandinas, 50, 195-213.

\section{Notas}

1 Según datos de Extranjería entre el 2008 y 2013 los grupos que han aumentado notoriamente en solicitudes de visas temporales, fuera de los de origen fronterizo, son los españoles, colombianos, dominicanos y haitianos. Por ejemplo en el caso de los españoles los permisos de residencia temporal pasaron de 712 el 2008 a 4.918 el 2013 y en el caso de los colombianos el aumento fue de 4.389 a 26.627 solicitudes para los mismos años (www.extranjería.gob.cl). Entre el 2014 y 2015 crece de manera significativa el otorgamiento de visas entre "los originarios de Venezuela (97\%), Cuba (77\%), Italia (63\%), Colombia (56\%), Haití (55\%), España (50\%), Dominicana (49\%) y Bolivia (43\%)" (Rojas y Silva 2016, p. 22).

2 Acepción que se utiliza para denominar "a las personas que viven en un país y que cruzan a trabajar a otro" (Castillo
Tapia, M. y Chacón, F.

2016 Vínculos transfronterizos: vida, movilidad y comercio en el Barrio Boliviano de Iquique, Chile. REMHU, Revista Interdisciplinar de la Movilidad Humana, 24, 131-152.

Tapia, M.; Liberona, N. y Contreras, Y.

2017 El surgimiento de un territorio circulatorio en la frontera chileno-peruana: estudio de las prácticas socioespaciales fronterizas. Geografía Norte Grande 66, 117-141.

Zapata-Barrero, R. y Ferrer-Gallardo, X.E.

2012 Fronteras en movimiento. Migraciones hacia la Unión Europea en el contexto Mediterráneo. Barcelona: Edicions Bellaterra.

Zimmerbauer, K.

2011 Conceptualizing Borders in Cross-Border Regions: Case Studies of the Barents and Ireland-Wales Supranational Regions. Journal of Borderlands Studies, 26(2), 211-229.

y Nájera 2014, p. 19) o para designar a las personas que cruzan la frontera (en ambos sentidos) con cualquier fin específico: trabajar, estudiar, visitar familiares, ir de turismo y de compras o quienes se mueven entre dos localidades en un mismo país (Chavez 2016).

3 En http://www.inearicayparinacota.cl/. Sitio web consultado el 7 de septiembre del 2016.

$4 \quad$ En http://priem.cl/wp-content/uploads/2015/04/Stefoni_ Perfil-Migratorio-de-Chile.pdf. Sitio web consultado el 8 de octubre 2016.

5 En http://www.bcrp.gob.pe/docs/Sucursales/Arequipa/ arequipa-caracterizacion.pdf. Sitio web revisado el 10 de octubre del 2016. 
